\title{
Participação nas aulas de educação física e indicadores de atitudes relacionadas à atividade física em adolescentes
}

CDD. 20.ed. 152.3

796.01

\author{
Carla Menêses HARDMAN* \\ Simone Storino Honda BARROS* \\ Maria Laura Siqueira de Souza ANDRADE****** \\ Juarez Vieira do NASCIMENTO* \\ Markus Vinicius NAHAS* \\ Mauro Virgílio Gomes de BARROS ${ }^{* / * * * * * *}$
}

*Centro de Desportos, Universidade Federal de Santa Catarina

**Escola Superior de Educação Física,

Universidade de Pernambuco.

***Departamento de Educação Física, Universidade Federal da Paraíba.

\section{Resumo}

0 objetivo deste estudo foi analisar a associação entre participação nas aulas de educação física (PAEF) e atitudes relacionadas à atividade física (gostar de fazer atividade física e preferir atividades de lazer fisicamente ativas) em adolescentes. Trata-se de um estudo epidemiológico transversal, a partir de uma amostra representativa de adolescentes ( $n=4.207,14-19$ anos) estudantes do ensino médio da rede pública estadual de Pernambuco. Dados foram coletados através de um questionário previamente validado. Observou-se que entre os adolescentes que relataram participar das aulas de educação física a chance de gostar de fazer atividade física foi 73\% maior nos rapazes e 93\% superior nas moças em comparação àqueles que não participam das aulas. A chance de preferir atividades de lazer fisicamente ativas foi $97 \% \mathrm{e}$ $72 \%$ superior nos rapazes e nas moças que PAEF, respectivamente. Concluiu-se que a PAEF está diretamente associada aos indicadores que expressam atitudes dos adolescentes em relação à atividade física.

Palavras-chave: Atividade motora; Educação física - Treinamento; Atividades de lazer; Adolescente; Estudantes.

\section{Introdução}

A formação e o desenvolvimento de atitudes em relação à atividade física $(\mathrm{AF})$ é um processo psicológico complexo que evolui continuamente para incluir as várias experiências de $\mathrm{AF}$ durante os anos na idade escolar ${ }^{1}$. A atitude é uma função da crença de que a participação na AF irá resultar em certos resultados, bem como a avaliação ou valor destes como tendo efeitos positivos ou negativos ${ }^{2-3}$. Neste sentido, alguns pesquisadores $^{4-5}$ afirmam que estudantes que tiveram experiências positivas em relação a prática de $\mathrm{AF}$ podem manter o interesse em realizá-la, por outro lado, os alunos que não se sentem sucedidos em uma atividade possivelmente não terão prazer em praticá-la.

Neste contexto, a participação nas aulas de educação física (PAEF) pode influenciar na prática de AF fora da escola, oferecendo oportunidades aos alunos de experimentar e de apreciar um estilo de vida fisicamente ativo ${ }^{6-9}$. Levantamento realizado por STELZER et al. ${ }^{1}$, com 1.107 estudantes do ensino médio de quatro países (Áustria, República Checa, Inglaterra e EUA), indicou que as atitudes dos estudantes dos Estados Unidos em relação às aulas de Educação Física (EF) foram mais negativas do que a de seus pares. Estudo longitudinal, conduzido por KJØNNIKSEN et $\mathrm{al}^{10}{ }^{10}$, com 630 adolescentes noruegueses, acompanhados durante 10 anos, indicou que as atitudes em relação às aulas de EF foram moderadamente relacionadas com a participação dos jovens em práticas esportivas durante a adolescência.

Outras investigações internacionais também indicaram que a prática de AF começa a declinar no período da adolescência ${ }^{11-13}$, por isso, é importante 
compreender as atitudes e as percepçóes dos estudantes do ensino médio em relação à atividade fisica, uma vez que, fatores demográficos, socioeconômicos, ambientais, socioculturais, comportamentais e psicognitivos podem influenciar a decisão de um indivíduo de iniciar ou continuar a participar em uma atividade ${ }^{12,14-15}$

Uma das estratégias adotadas para esclarecer porque os indivíduos estão expostos a níveis insuficientes de AF é o desenvolvimento de estudos

\section{Método}

Para desenvolvimento deste estudo transversal, recorreu-se à análise secundária dos dados de um levantamento epidemiológico de abrangência estadual e base escolar, intitulado "Estilos de Vida e Comportamentos de Risco à Saúde em Estudantes do Ensino Médio no Estado de Pernambuco".

O protocolo de investigação que derivou os dados para realização deste estudo foi aprovado pelo Comitê de Ética em Pesquisa com Seres Humanos do Hospital Agamenon Magalhães (Recife - PE, Brasil). Todas as diretrizes estabelecidas nas resoluçōes $196 \mathrm{e}$ 251, do Conselho Nacional de Saúde, foram observadas no desenvolvimento deste estudo. Um termo negativo de consentimento foi usado para obter dos pais ou responsáveis pelos estudantes menores de 18 anos permissão para que os mesmos participassem do estudo. A participação de todos os sujeitos foi voluntária e todos foram informados de que poderiam desistir em qualquer etapa da fase de coleta de dados.

A população alvo foi limitada aos estudantes do ensino médio da rede pública estadual de Pernambuco, com idade entre 14 e 19 anos. Considerando-se todas as dependências administrativas (federal, estadual, municipal e privada), os estudantes matriculados na rede pública estadual representavam na época (2006) aproximadamente $80 \%$ do total em todo Estado.

O dimensionamento amostral foi efetuado de forma a atender os diversos objetivos do projeto, que incluíam a avaliação da exposição a 10 fatores comportamentais de risco à saúde, além de medidas antropométricas e da pressão arterial em repouso. Para o cálculo do tamanho amostral foram adotados os seguintes parâmetros: população estimada em 353 mil estudantes; intervalo de confiança de 95\%; poder estatístico de $80 \%$; erro máximo tolerável de três pontos percentuais; e a prevalência estimada foi arbitrada em 50\%. visando identificar atitudes relacionadas à $\mathrm{AF}$. Entretanto, pouco se conhece sobre os indicadores que expressam atitudes relacionadas à $\mathrm{AF}$ em adolescentes da região nordeste do Brasil. A avaliação destes indicadores é um procedimento importante para conhecer o interesse desta população e orientar o planejamento de programas de intervenção adequados. Nesta perspectiva, o presente estudo teve como objetivo analisar a associação entre a PAEF e atitudes relacionadas à $\mathrm{AF}$ em adolescentes.

Recorrendo-se a cálculos de poder estatístico "a posteriori”, verificou-se que este dimensionamento amostral permitiria efetuar análise de associação entre as variáveis com possibilidade de detectar como estatisticamente significativas razões de "odds" (OR) de 1,3 ou superiores, considerando-se: prevalência de gostar de fazer AF entre $72 \%$ a $83 \%$ nos não expostos e entre $76 \%$ a $86 \%$ nos expostos; prevalência de ter preferências pelas atividades de lazer fisicamente ativas entre $36 \%$ a $50 \%$ nos não expostos e entre $41 \%$ a $54 \%$ nos expostos; poder estatístico de $80 \%$; e nível de confiança de $95 \%$. Cálculos amostrais e de poder estatístico foram efetuados conforme recomendado em KeLSEY et al. ${ }^{16}$.

Procurou-se garantir que a amostra selecionada representasse a população alvo considerando a sua distribuição conforme região geográfica, período de matrícula (diurno e noturno) e porte das escolas (pequenas, com menos de 200 alunos; médias, com 200 a 499 alunos; e, grandes, com 500 estudantes ou mais). Alunos matriculados no período da manhã e da tarde foram agrupados numa única categoria (estudantes do período diurno). A distribuição regional foi observada pelo número de escolas existentes em cada uma das 17 Gerências Regionais de Ensino da Secretaria de Educação do Estado.

Para seleção da amostra, recorreu-se a um procedimento de amostragem por conglomerados em dois estágios, sendo que a "escola" e a "turma" representaram, respectivamente, as unidades amostrais no primeiro e no segundo estágio. Todas as escolas da rede pública estadual que ofereciam ensino médio regular foram consideradas elegíveis para inclusão no estudo. No primeiro estágio, adotou-se como critério de estratificação a densidade de escolas em cada microrregião do Estado segundo porte das mesmas, assim foram sorteadas, proporcionalmente, mais escolas nas 
microrregiōes onde a densidade era também maior. No segundo estágio, considerou-se a densidade de turmas nas escolas sorteadas por período de assistência às aulas como critério para sorteio daquelas nas quais os questionários seriam aplicados. Todos os alunos das turmas sorteadas foram convidados a participar do estudo, independente da idade dos mesmos. Após a aplicação, os questionários respondidos por alunos com idade superior a 19 anos e aqueles com idade inferior a 14 anos foram excluídos.

O período de realização do estudo foi de abril a outubro de 2006. A coleta dos dados foi realizada por equipe previamente treinada, composta por seis estudantes de pós-graduação, seguindo um protocolo padronizado de coleta de dados. A aplicação dos questionários foi efetuada em sala de aula e os sujeitos foram continuamente assistidos por dois aplicadores que esclareciam as dúvidas e auxiliavam no preenchimento das informações.

O questionário utilizado foi uma versão traduzida e adaptada do Global School-based Student Health Survey, proposto pela Organização Mundial da Saúde, em colaboração com outras entidades ${ }^{17}$. O instrumento foi validado (validade de conteúdo e de face) e previamente testado em duas escolas da rede municipal de ensino na cidade do Recife, sendo administrado na forma de entrevista em pequenos grupos. Indicadores de reprodutibilidade (consistência de medidas teste-reteste) foram de moderados a altos na maioria dos itens do instrumento, sendo que os coeficientes de concordância (índice kappa) variaram de 0,52 a 1,00 .

Todas as informações obtidas neste estudo foram autorreferidas. As variáveis dependentes foram os indicadores que expressam atitudes em relação à $\mathrm{AF}$. Estes dados foram extraídos de duas perguntas: "Eu gosto de fazer AF! O que você diria desta afirmação?" e "Qual a atividade de lazer de sua preferência?".

A avaliação da afirmação referente a gostar de fazer AF foi efetuada considerando cinco opções

\section{Resultados}

Do total de adolescentes presentes nas turmas sorteadas nas 76 escolas investigadas (4.269), 55 estudantes se rejeitaram a participar e sete foram excluídos devido a dados incompletos no questionário. de resposta em escala tipo Likert. Posteriormente, esta variável foi categorizada em duas categorias: discorda (discordo totalmente, discordo em partes, nem concordo, nem discordo) e concorda (concordo em parte, concordo totalmente). A preferência de atividades de lazer também foi agrupada em duas categorias: "atividades de lazer fisicamente ativas" (esportes, exercícios, nadar, pedalar) e "atividades de lazer sedentárias" (jogar dominó ou cartas, assistir $\mathrm{TV}$, jogar videogame, usar o computador, conversar com os amigos).

A variável independente foi à PAEF. Os sujeitos foram classificados em dois grupos (participantes/ não participantes), sendo que os "participantes" foram aqueles que relataram participar em pelo menos uma aula de EF por semana. Considerou-se como potenciais fatores de confusão as seguintes variáveis: faixa etária (14-16 anos, 17-19 anos), local de residência (urbana, rural), situação ocupacional (trabalha, não trabalha) e turno escolar (diurno, noturno).

Para realização das análises, utilizou-se Data Analysis and Statistical Software (STATA), versão 10, empregando-se procedimentos de estatística descritiva (distribuição de frequência, média, desvio padrão) e indutiva. Para comparar a prevalência de adolescentes que relataram gostar de fazer $\mathrm{AF}$ e preferir atividades de lazer fisicamente ativas em função das categorias da variável independente (PAEF) recorreu-se aplicação do teste de Qui-quadrado.

Análises de associação entre a variável independente e as dependentes foram conduzidas mediante utilização de regressão logística separadamente para cada uma das variáveis dependentes. Inicialmente, procederam-se análises brutas e, em seguida, as análises multivariáveis que foram conduzidas a fim de controlar potenciais fatores de confusão, sendo que tanto as análises brutas quanto as ajustadas foram estratificadas por sexo devido à identificação de interação.

A amostra final ficou constituída por 4.207 adolescentes (59,8\% do sexo feminino). A média de idade dos sujeitos foi 16,8 $\pm 1,4$ anos. A TABELA 1 apresenta as características sociodemográficas da amostra por sexo. 
TABELA 1 - Características sociodemográficas da amostra por sexo.

Dados faltantes:

\begin{tabular}{|c|c|c|c|c|c|c|}
\hline \multirow{2}{*}{ Variável } & \multicolumn{2}{|c|}{ Rapazes } & \multicolumn{2}{|c|}{ Moças } & \multicolumn{2}{|c|}{ Todos } \\
\hline & $\mathbf{n}$ & $\%$ & $\mathbf{n}$ & $\%$ & $\mathbf{n}$ & $\%$ \\
\hline \multicolumn{7}{|l|}{ Faixa etária* } \\
\hline $14-16$ anos & 598 & 35,4 & 1.165 & 46,4 & 1.766 & 42,0 \\
\hline 17-19 anos & 1.089 & 64,6 & 1.346 & 53,6 & 2.441 & 58,0 \\
\hline \multicolumn{7}{|l|}{ Local de residência ${ }^{¥}$} \\
\hline Urbana & 1.311 & 78,1 & 1.983 & 79,5 & 3.294 & 79,0 \\
\hline Rural & 367 & 21,9 & 510 & 20,5 & 877 & 21,0 \\
\hline \multicolumn{7}{|l|}{ Situação ocupacional ${ }^{\$}$} \\
\hline Não trabalha & 1.157 & 69,2 & 2.119 & 84,8 & 3.276 & 78,5 \\
\hline Trabalha & 514 & 30,8 & 381 & 15,2 & 895 & 21,5 \\
\hline \multicolumn{7}{|l|}{ Turnos } \\
\hline Diurno (manhã/tarde) & 908 & 53,9 & 1.506 & 60,0 & 2.414 & 57,6 \\
\hline Noturno & 778 & 46,1 & 1.002 & 40,0 & 1.780 & 42,4 \\
\hline
\end{tabular}

Em relação aos indicadores que expressam atitudes relacionadas à AF, 76,3\% (IC95\%: 75,0-77,6) dos adolescentes referiram gostar de fazer $\mathrm{AF}$ e $41,5 \%$ (IC95\%: 39,9-43,1) relataram preferir atividades de lazer fisicamente ativas. As atividades de lazer preferidas dos estudantes foram: esportes $(25,0 \%)$, conversar com os amigos (23,5\%) e assistir TV (18,7\%).

A proporção de adolescentes que relataram gostar de fazer AF foi significativamente maior $(\mathrm{p}<0,001)$ entre os rapazes (83,7\%; IC95\%: 82,5-84,8) em comparação as moças (71,4\%; IC95\%: 70,0-72,8). De modo similar, a distribuição dos sujeitos que referiram preferir atividades de lazer fisicamente ativas foi estatisticamente superior $(\mathrm{p}<0,001)$ entre os rapazes $(66,9 \%$; IC95\%: 65,4-68,4) em comparação as moças (23,2\%; IC95\%: 21,9-24,6). Na TABELA 2 está descrita a distribuição dos sujeitos que relataram gostar de fazer $\mathrm{AF}$ e preferir atividades de lazer fisicamente ativas segundo categorias das variáveis sociodemográficas.

TABELA 2 - Proporção de adolescentes que referiram "gostar de fazer AF" e "preferir atividades de lazer fisicamente ativas” de acordo com variáveis sociodemográficas. Pernambuco, Brasil, 2006.

\begin{tabular}{|c|c|c|c|c|}
\hline \multirow{2}{*}{ Variável } & \multicolumn{2}{|c|}{ Gostar de fazer AF } & \multicolumn{2}{|c|}{ Preferir atividades de lazer fisicamente ativas } \\
\hline & n & $\%$ & $\mathbf{n}$ & $\%$ \\
\hline \multicolumn{5}{|l|}{ Faixa etária } \\
\hline $14-16$ anos & 1.335 & 75,9 & 607 & 38,3 \\
\hline 17-19 anos & 1.867 & 76,6 & 937 & 43,8 \\
\hline Valor de $\mathrm{p}$ & 0,575 & & 0,001 & \\
\hline \multicolumn{5}{|l|}{ Local de residência } \\
\hline Urbana & 2.515 & 76,5 & 1.196 & 41,2 \\
\hline Rural & 665 & 75,6 & 337 & 42,5 \\
\hline Valor de p & 0,615 & & 0,506 & \\
\hline \multicolumn{5}{|l|}{ Situação ocupacional } \\
\hline Não trabalha & 2.474 & 75,6 & 1.140 & 39,1 \\
\hline Trabalha & 710 & 79,2 & 392 & 50,1 \\
\hline Valor de $\mathrm{p}$ & 0,023 & & $<0,001$ & \\
\hline \multicolumn{5}{|l|}{ Turno } \\
\hline Diurno (manhā/tarde) & 1.838 & 76,3 & 838 & 38,8 \\
\hline Noturno & 1.361 & 76,4 & 704 & 45,2 \\
\hline Valor de p & 0,909 & & $<0,001$ & \\
\hline \multicolumn{5}{|l|}{ Excesso de peso } \\
\hline Não & 2.841 & 76,5 & 1.365 & 41,4 \\
\hline Sim & 361 & 74,7 & 179 & 41,9 \\
\hline Valor de $\mathrm{p}$ & 0,383 & & 0,845 & \\
\hline
\end{tabular}

626 • Rev Bras Educ Fís Esporte, (São Paulo) 2013 Out-Dez; 27(4):623-31 
Verificou-se que a proporção de adolescentes que referiram gostar de fazer $\mathrm{AF}$ e preferir atividades de lazer fisicamente ativas estão estatisticamente associados à PAEF $(p<0,001)$. A FIGURA 1 apresenta os indicadores que expressam atitudes relacionadas à $\mathrm{AF}$ associados à PAEF, estratificado por sexo.
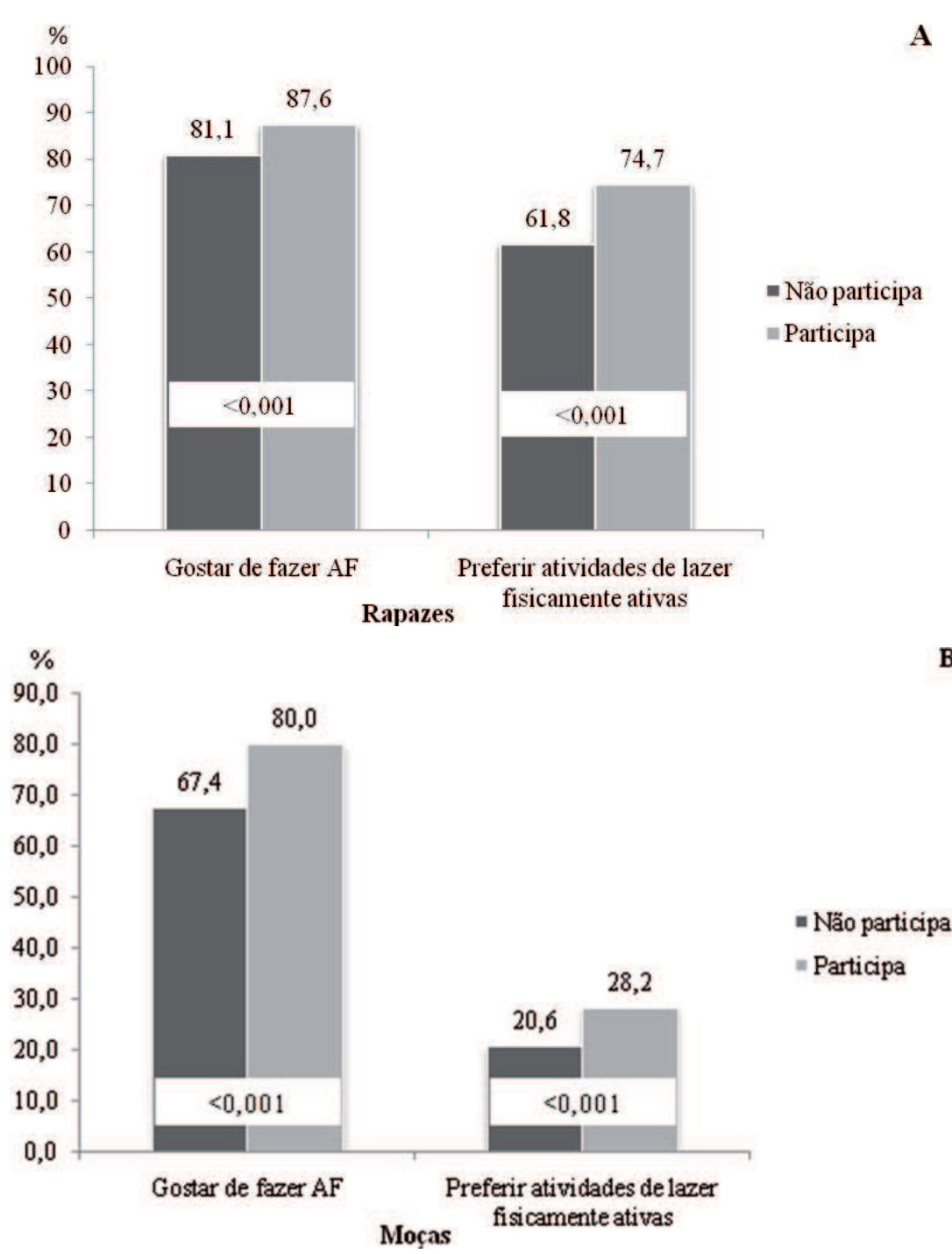

FIGURA 1 - Proporção de rapazes (A) e moças (B) que referiram "gostar de fazer AF" e "preferir atividades de lazer fisicamente ativas” de acordo com a PAEF. Pernambuco, Brasil, 2006.

Após ajustamento para variáveis de confusão, verificou-se que, em ambos os sexos, a PAEF está diretamente associada aos indicadores que expressam atitudes dos adolescentes em relação à $\mathrm{AF}$ (TABELAS 3 e 4). Observou-se que entre os adolescentes que participam de, no mínimo, uma aula por semana, a chance de gostar de fazer $\mathrm{AF}$ foi $73 \%$ maior nos rapazes e $93 \%$ superior nas moças em comparação àqueles que não participam das aulas. A chance de preferir atividades de lazer fisicamente ativas foi $97 \%$ e $72 \%$ superior nos rapazes e nas moças que PAEF, respectivamente. 
TABELA 3 - Análise bruta e ajustada para associação entre PAEF e gostar de fazer AF em adolescentes do ensino médio de Pernambuco, 2006. * Ajustado para variá-
veis: faixa etária, local
de residência, situação
ocupacional, turno es-
colar.

\begin{tabular}{lccccc}
\hline \multirow{2}{*}{ Variável } & \multicolumn{5}{c}{ Gostar de fazer AF } \\
\cline { 2 - 6 } & Categorias & OR bruto & $\mathbf{p}$ & OR ajustado* & p \\
\hline PAEF (rapazes) & Não & 1 & & 1 & \\
& Sim & $1,65(1,24-2,21)$ & $<0,001$ & $1,73(1,29-2,32)$ & $<0,001$ \\
\multirow{2}{*}{ PAEF (moças) } & Não & 1 & & 1 & \\
& Sim & $1,94(1,58-2,38)$ & $<0,001$ & $1,93(1,53-2,45)$ & $<0,001$ \\
\hline
\end{tabular}

TABELA 4 - Análise bruta e ajustada para associação entre PAEF e preferir atividades de lazer fisicamente ativas em adolescentes do ensino médio de Pernambuco, 2006.

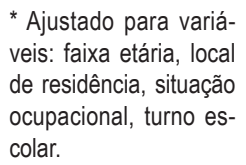

\begin{tabular}{lccccc}
\hline \multirow{2}{*}{ Variável } & \multicolumn{5}{c}{ Preferir atividades de lazer fisicamente ativas } \\
\cline { 2 - 6 } & Categorias & OR bruto & $\mathbf{p}$ & OR ajustado* & p \\
\hline PAEF (rapazes) & Náo & 1 & & 1 & \\
& Sim & $1,82(1,45-2,30)$ & $<0,001$ & $1,97(1,60-2,43)$ & $<0,001$ \\
\multirow{2}{*}{ PAEF (moças) } & Não & 1 & & 1 & \\
& Sim & $1,51(1,22-1,87)$ & $<0,001$ & $1,72(1,38-2,13)$ & $<0,001$ \\
\hline
\end{tabular}

\section{Discussão}

Os achados deste estudo revelaram que a PAEF é um fator diretamente associado aos indicadores que expressam atitudes relacionadas à $\mathrm{AF}$ (gostar de fazer AF e preferir atividades de lazer fisicamente ativas), independentemente do sexo. Este levantamento também indicou que gostar de fazer $\mathrm{AF}$ é uma atitude mais prevalente, relatada pelos estudantes pernambucanos, do que preferir atividades de lazer fisicamente ativa.

No presente estudo, verificou-se que a proporção de adolescentes que referiram tais atitudes foi significativamente maior entre os rapazes em comparação as moças. Este resultado é consistente com a evidência de que estudantes do sexo masculino nutrem uma atitude fortemente positiva em relação a $\mathrm{AF}^{1,18}$. MARZINEK ${ }^{18}$ sugere que os vínculos criados entre os rapazes durante as aulas de EF permanecem presentes em outras atividades do cotidiano. Além disso, este estudo apontou que os rapazes foram mais propensos a gostar e ter prazer em participar das aulas de EF.

Estudos prévios indicaram diferenças entre o sexo e a atitude relacionada a AF baseada no tipo de atividade ${ }^{19-22}$. Por exemplo, os rapazes apresentam atitudes mais positivas em relação às atividades físicas coletivas e aquelas que são desafiadoras, enquanto as moças apresentam atitudes favoráveis para atividades que enfatizam a estética ou movimentos graciosos.
Diferente do que observado em alguns estudos que investigaram as atitudes dos alunos em relação à idade ${ }^{23-24}$, no presente estudo, verificou-se que a proporção de adolescentes que relataram atitudes positivas relacionadas a $\mathrm{AF}$ foi maior nos adolescentes mais velhos. Uma explicação plausível para este achado pode estar relacionada a independência/ autonomia dos adolescentes para realizar atividades individuais e/ou aquelas que proporcionam desafios. Por exemplo, atividades com diferentes exigências físicas e cognitivas ou atividades que exijam habilidades ou desenhos ofensivos e estratégias defensivas podem despertar interesse entre os estudantes ${ }^{25}$.

Embora os esportes tenham sido a atividade de lazer preferida pela maioria dos adolescentes, constatou-se que $58,5 \%$ dos estudantes relataram preferência por atividades sedentárias (jogar dominó ou cartas, assistir TV, jogar videogame, usar o computador, conversar com os amigos). Certos fatores contribuem para limitar a participação dos adolescentes em jogos e esportes em seu tempo livre, tais como as obrigações escolares e de formação profissional, as tarefas do lar e o precoce ingresso no mercado de trabalho. Segundo SARRIERA et al. ${ }^{26}$, a forma como é vivenciado o lazer parece ter forte ligação com a estrutura social a qual pertence o indivíduo, levando-se em consideração aspectos da sua conjuntura social, cultural, econômica 
e ideológica. No presente estudo, verificou-se que o sexo, a faixa etária, a situação ocupacional e o turno escolar dos sujeitos parecem estar associados às preferências por atividades de lazer.

As preferências de atividades ativas no lazer têm influência positiva na adoção de um estilo de vida fisicamente ativo e na qualidade de vida das pessoas ${ }^{27}$. A identificação prévia das atividades realizadas no contexto do lazer é um aspecto importante para o planejamento e a implementação de intervenções para promoção de AF.

De forma semelhante, estudos têm sugerido que as aulas de EF têm um grande potencial de promover conhecimentos e atitudes positivas em relação à saúde ${ }^{28-30}$. Para alguns estudantes, a EF é a única oportunidade onde eles se envolvem em $\mathrm{AF}^{31}$. Neste sentido, atitudes positivas adquiridas nas aulas de EF podem desempenhar um papel importante na manutenção de um estilo de vida fisicamente ativo ${ }^{10}$.

No entanto, algumas limitaçôes precisam ser consideradas neste estudo. Trata-se de um levantamento de base escolar delimitado aos adolescentes do ensino médio matriculados em escolas públicas estaduais, aspecto que não permite extrapolar os resultados deste estudo para toda população adolescente. Por outro lado, os dados foram obtidos mediante utilização de um questionário que foi previamente testado e apresentou boa consistência intra-avaliador. Outro ponto positivo refere-se à utilização de dois indicadores (gostar de fazer $\mathrm{AF}$ e preferências de atividades físicas no lazer) que apresentam os principais componentes (prazer e utilidade percebida) de como as atitudes são formadas. Estes componentes abrangem tanto domínio afetivo quanto cognitivo e têm sido utilizados como o arcabouço teórico ${ }^{24,32}$.

Portanto, o oferecimento de atividades prazerosas aos adolescentes pode contribuir para o aumento da adesão a $\mathrm{AF}$ e atitudes positivas ${ }^{1}$. HAGGER et al. ${ }^{33}$ também mencionam que a criação de um ambiente no qual o adolescente se sente confortável e confiante irá reforçar atitudes positivas.

Conclui-se que a participação nas aulas de Educação Física está diretamente associada aos indicadores que expressam atitudes relacionadas à AF (gostar de fazer $\mathrm{AF}$ e preferir atividades de lazer fisicamente ativas) em adolescentes pernambucanos. Os resultados do presente estudo indicam que a participação nas aulas de Educação Física pode ter papel importante na promoção da atividade física, tendo em vista a sua associação com fatores mediadores desta conduta, a exemplo das atitudes dos estudantes adolescentes.

$\mathrm{O}$ acesso a informaçóes e as experiências que podem ser vivenciadas nas aulas de Educação Física podem concorrer para atitudes mais positivas e, por conseguinte, para maior nível de prática de atividades físicas, mas esta hipótese causal precisa ser testada em futuras investigações.

A compreenção das atitudes (crenças e sentimentos) dos adolescentes em relação à $\mathrm{AF}$ é importante e merece atenção dos pais, professores e pesquisadores. Futuros estudos deverão verificar se há relação causal entre estes fatores e apresentar possíveis explicaçōes para estes achados. Investigações posteriores, com abordagem qualitativa, também poderão explorar as atitudes e intenções dos adolescentes como um constructo multidimensional.

\begin{abstract}
Participation in physical education classes and indicators of attitudes toward physical activity in adolescents

The objective of this study was to analyze the association between the participation in physical education classes (PPEC) and attitudes towards physical activity (to enjoy physical activity and to prefer active leisure activities) in adolescents. This is a cross-sectional epidemiological study from a representative sample of adolescents ( $n=4207,14-19$ years) high school students of public schools from Pernambuco. Data were collected using a previously validated questionnaire. It was found that among adolescents who reported PPEC the chance to enjoy physical activity was 73\% higher in boys and 93\% higher in girls compared to those who do not participate in the classes. The chance of to prefer active leisure activities was $97 \%$ and $72 \%$ higher in boys than in girls and PPEC, respectively. It was concluded that the PPEC is directly associated with the indicators that express attitudes of adolescents in relation to physical activity.
\end{abstract}

KEY woRDS: Motor activity; Physical education - training; Leisure activities; Adolescent; Students. 


\section{Referências}

1. Stelzer J, Ernest JM, Fenster MJ, Langford G. Attitudes toward physical education: a study of high school students from four countries - Austria, Czech Republic, England, and USA. Coll Stud J. 2004;38:171-8.

2. Deforche BI, Bourdeaudhuij IM, Tanghe AP. Attitude toward physical activity in normal-weight, overweight and obese adolescents. J Adolesc Health. 2006;38:560-8.

3. Bernstein E, Phillips SR, Silverman S. Attitudes and perceptions of middle school students toward competitive activities in physical education. J Teach Phys Educ. 2011;30:69-83.

4. Subramaniam PR, Silverman S. Validation of scores from an instrument assessing student attitude toward physical education. Meas Phys Educ Exerc Sci. 2000;4:29-43.

5. Caetano A, Januário C. Motivação, teoria das metas discentes e competência percebida. Pensar Prát. 2009;12.

6. Ommundsen Y. Implicit theories of ability and self-regulation strategies in physical education classes. Educ Psychol: Int J Exp Educ Psychol. 2003;23:141-57.

7. Portman PA. Are physical education classes encouraging students to be physically active? Experience of ninth graders in their last semester of required physical education. Phys Educ. 2003;3:150-61.

8. Trudeau F, Shephard RJ. Contribution of school programmes to physical activity levels and attitudes in children and adults. Sports Med. 2005;2:89-105.

9. US Department of Health and Human Services. Physical activity guidelines advisory committee report, 2008. Washington: DHHS; 2008.

10. Kjønniksen L, Wold B, Fjørtoft I. Attitude to physical education and participation in organized youth sports during adolescence related to physical activity in young adulthood: a 10-year longitudinal study. Eur Phys Educ Rev. 2009;15:139-54.

11. Caspersen CJ, Pereira MA, Curran KM. Changes in physical activity patterns in the United States, by sex and crosssectional age. Med Sci Sports Exerc. 2000;32:1601-9.

12. Sallis JF, Prochaska JJ, Taylor WC. A review of correlates of physical activity of children and adolescents. Med Sci Sports Exerc. 2000;5:963-75.

13. Allison KR, Adlaf EM, Dwyer JJM, Lysy DC, Irving HM. The decline in physical activity among adolescent students: a cross-national comparison. Can J Public Health. 2007;98:97-100.

14. Bibik J, Orsega-Smith E, Goodwin S. High school students' attitudes towards physical education in Delaware. Phys Educ. 2007;64:192-204.

15. Truccolo AB, Maduro PA, Feijó EA. Fatores motivacionais de adesão a grupos de corrida. Motriz. 2008;14:108-14.

16. Kelsey JL, Whittemore AS, Evans AS, Thompson WD. Methods in observational epidemiology. 2nd ed. New York: Oxford University Press; 1996.

17. World Health Organization. Global School-Based Student Health Survey. Atlanta: World Health Organization [citado 1 abr. 2013]. Disponível em: http://www.cdc.gov/GSHS/.

18. Marzinek A. A motivação de adolescentes nas aulas de educação física [dissertação]. Brasília(DF): Universidade Católica de Brasília; 2004.

19. Hicks MK, Wiggins MS, Crist RW, Moode FM. Sex differences in grade three students' attitudes toward physical activity. Percept Mot Skills. 2001;93:97-102.

20. Salles-Costa R, Heilborn ML, Werneck GL, Faerstein E, Lopes CS. Gênero e prática de atividade física de lazer. Cad Saude Publica. 2003;19:S325-33.

21. Vilhjalmsson R, Kristjansdottir G. Gender differences in physical activity in older children and adolescents: the central role of organized sport. Soc Sci Med. 2003;56:363-74.

22. Slater A, Tiggemann M. Gender differences in adolescent sport participation, teasing, self-objectification and body image concerns. J Adolesc. 2011;34:455-63.

23. Wersch AV, Trew K, Turner I. Post-primary school pupils' interest in physical education: age and gender differences. Br J Educ Psychol. 1992;62:56-72.

24. Prochaska JJ, Sallis JF, Slymen DJ, Mckenzie TL. A longitudinal study of children's enjoyment of physical education. Pediatr Exerc Sci. 2003;15:170-8.

25. Chen A, Darst PW. Situational interest in physical education: A function of learning task design. Res Q Exerc Sport. 2001;72:150-64.

26. Sarriera JC, Tatim DC, Coelho RPS, Büsker J. Uso do tempo livre por adolescentes de classe popular. Psicol Reflex Crít. 2007;20:361-7. 
27. Pylro SC, Rossetti CB. Atividades lúdicas, gênero e vida adulta. Psico USF. 2005;10:77-86.

28. Brasil. Ministério da Educação e Cultura, Secretaria de Educação Média e Tecnológica. Parâmetros Curriculares Nacionais - Ensino Médio. Brasília: MEC; 2000.

29. Terres NG, Pinheiro RT, Horta BL, Pinheiro KAT, Horta LL. Prevalência e fatores associados ao sobrepeso e à obesidade em adolescentes. Rev Saude Publica. 2006;4:627-33.

30. Brasil. Ministério da Saúde. Secretaria de Atenção à Saúde. Departamento de Atenção Básica. Saúde na Escola. Brasília (DF): Ministério da Saúde (Série B. Textos Básicos de Saúde). Cad Atenção Básica. 2009;24:96.

31. Nahas MV. Atividade física, saúde e qualidade de vida: conceitos e sugestôes para um estilo de vida ativo. 5a ed. Londrina: Midiograf; 2010.

32. Subramaniam PR, Silverman S. Middle school students' attitudes toward physical education. Teach Teach Educ. 2007;23:602-11.

33. Hagger MS, Chatzisarantis NL, Biddle JH. A meta-analytic review of the theories of reasoned action and planned behavior in physical activity: Predictive validity and the contribution of additional variables. J Sport Exerc Psychol. 2002;24:3-32.

\section{Agradecimentos}

Os pesquisadores agradecem à Secretaria de Educação e Cultura de Pernambuco e às Gerências Regionais de Educação, pelo apoio logístico. Aos gestores e docentes das escolas que contribuíram para a execução da coleta dos dados e aos discentes que participaram do estudo. Aos integrantes do Grupo de Pesquisa em Estilos de Vida e Saúde da Escola Superior de Educação Física da Universidade de Pernambuco que colaboraram na coleta e tabulação dos dados.

Este estudo contou com o apoio do CNPq pela concessão de auxílio financeiro a projeto de pesquisa (processo $\mathrm{n}^{\circ}$ 486023/2006-0) e bolsa de produtividade em pesquisa (processo $n^{\circ} 307415 / 2010-4$ ). FACEPE e CAPES pela concessão de bolsas de estudo e recursos de custeio para o desenvolvimento do projeto.

\begin{tabular}{r|r} 
ENDEREço & \\
Carla Menêses Hardman & \\
Universidade Federal de Santa Catarina & Recebido para publicação: 14/08/2012 \\
Centro de Desportos & Revisado: 05/04/2013 \\
Programa de Pós-Graduação em Educação Física & Aceito: 18/04/2013 \\
Campus Universitário - Trindade. & \\
88040-900 - Florianópolis - SC - BRASIL & \\
e-mail: carlinhams@gmail.com &
\end{tabular}

